\title{
A Formalism for Utilization of Sensor Systems and Integrated Project Models for Active Construction Quality Control
}

\author{
Burcu Akinci ${ }^{1}$, Frank Boukamp ${ }^{2}$, Chris Gordon ${ }^{3}$, Daniel Huber ${ }^{4}$, Catherine Lyons ${ }^{5}$, Kuhn Park ${ }^{6}$ \\ Carnegie Mellon University
}

\begin{abstract}
Defects experienced during construction are costly and preventable. However, inspection programs employed today cannot adequately detect and manage defects that occur on construction sites, as they are based on measurements at specific locations and times, and are not integrated into complete electronic models. Emerging sensing technologies and project modeling capabilities motivate the development of a formalism that can be used for active quality control on construction sites. In this paper, we outline a process of acquiring and updating detailed design information, identifying inspection goals, inspection planning, as-built data acquisition and analysis, and defect detection and management. We discuss the validation of this formalism based on four case studies.
\end{abstract}

Keywords: Quality control, construction inspection, sensors, laser scanners, integrated project models, object recognition

\section{Introduction}

Current approaches for quality control on construction sites are not as effective as they could be in identifying defects early in the construction process. As a result, defects can go undetected until later phases of construction or even to the maintenance phase. Defects, when detected late, can have costly ramifications. It has been noted that six to twelve percent of construction cost is wasted due to rework of defective components detected late in the construction phase [1][2][3] and five percent of construction cost is wasted due to rework of defective components detected during maintenance [3]. Twenty to forty percent of all these site defects can be attributed to the construction phase [3].

\footnotetext{
${ }^{1}$ Corresponding Author; Assist. Prof., Civil and Environmental Engineering, Carnegie Mellon University, Pittsburgh, PA 15213, bakinci@cmu.edu

${ }^{2} \mathrm{PhD}$ Candidate, Civil and Environmental Engineering, Carnegie Mellon University

${ }^{3} \mathrm{PhD}$ Candidate, Civil and Environmental Engineering, Carnegie Mellon University

${ }^{4}$ Research Scientist, Robotics Institute, Carnegie Mellon University.

${ }^{5}$ Research Scientist, Robotics Institute, Carnegie Mellon University.

${ }^{6} \mathrm{PhD}$ Candidate, Department of Architecture, Carnegie Mellon University.
} 
According to [4], fifty-four percent of all construction defects are related to human factors such as unskilled workers or insufficient supervision of construction work. Furthermore, twelve percent of construction defects are due to material and system failures [4]. These statistics all suggest the importance of inspection for achieving higher construction quality. In addition, it has also been identified that careful inspection during construction is one of the most important factors in preventing structural failures during construction [5].

The status of the work in place at construction sites changes continuously as construction projects evolve over time. Current surveying and quality control approaches are not effective, since they only provide data at specific locations and times to represent the work in place, and the data generated are interpreted manually and are not integrated electronically into the project design and schedule. Consequently, project managers do not get complete and accurate information about work in place. This limits their abilities to easily identify and manage defects, and actively control and manage construction projects. Frequent, complete, and accurate assessment of the status of as-built conditions at construction sites, identification of critical spatio-temporal and material quality related deviations of work in place, and assessment of whether these deviations constitute defects during a construction project are necessary for active project quality control.

Recent advances in generating 3D environments using laser scanning technologies, and acquiring quality information about built environments using embedded and other advanced sensors create an opportunity to explore the technological feasibility of frequently gathering complete and accurate three-dimensional and material quality related as-built data. Laser scanners are being utilized to collect 3D geometric as-built information for renovation, retrofit and expansion projects in industrial, commercial and heavy-civil sectors of construction, and a set of these studies suggests some cost benefits of using scanners for quality control purposes [6][7][8]. Embedded sensing is also being adopted by various owners for assessment of quality of cast-inplace concrete. According to a survey of American state departments of transportation in 2000, thirty-two of the forty-four respondents indicated progress in investigating the sensor-based maturity method for their quality and project control purposes, and thirteen had already developed a protocol for its use [9].

Over a decade of research on integrated project models and current usage trends in the Architecture/Engineering/Construction (AEC) industry have shown that a semantically rich project data model, combining multiple project participants' views, can support various project management and facility management functions [10][11][12][13]. This provides an opportunity to integrate as-built data collected from laser scanners and embedded sensor systems with planned design and schedule models to assess whether there are important deviations between as-designed and as-built conditions.

Our research has shown that current sensing and scanning technologies and modeling approaches are technologically advanced, but the usage of them should be carefully planned and the data collected from them should be carefully analyzed in support of the desired task. There is a need for developing a formalism for utilizing these sensing and scanning technologies and for analyzing the data collected. Our approach includes utilization of prior design, schedule, and construction specification knowledge to determine what sensors to use when, and where to locate 
the sensors to enable efficient and effective data collection and to analyze the as-built data collected from these sensor systems to detect construction defects [14].

In this paper, we provide an overview of a formalism developed for active construction quality control using sensors and integrated project models. The focus of the formalism has been to leverage the technological advances in the automated data capture area. We have tested the technological aspects of this formalism on four construction job sites and in the last section of this paper, we describe our validation of the technical feasibility of the formalism by conducting retrospective and concurrent cases at these four sites.

\section{Background research}

Automated quality control approaches developed so far have mostly focused on streamlining the document management process associated with quality assurance and quality management on construction sites. Riley and Pickering have suggested an automated total quality management system composed of quality assurance process, form libraries and report management modules [15]. Similarly, two of the previous research projects focused on developing a computer-based implementation of ISO 9000 and 9001 guidelines on how to structure and store various qualityrelated information and documents such as inspection and test plans by utilizing templates generated by the systems developed [16][17]. These systems provide a formalism that enables contractors to implement ISO guidelines; however, they have not included an approach that leverages automated data capture technologies for creating and reasoning about as-built models during quality control processes.

Laser scanners are proving to be effective for capturing detailed 3D spatial data in order to create 3D as-built models. Cheok et al. demonstrated how LADAR (Laser Distance and Ranging) can be used for construction progress monitoring by focusing on and developing an approach for excavation work [18]. Their approach included generating and analyzing terrain models based on the data collected from LADARs to assess the progress of an excavation activity. Kwon et al. developed an approach that fits range data to geometric primitives so that $3 \mathrm{D}$ as-built modeling can be achieved rapidly [19]. This approach utilizes sparse range point clouds and targets increasing the efficiency of processing the data collected to enable real-time field applications. Kim et al. have developed a laser-based aggregate scanning technique utilizing scan data to automatically identify sizes of stone aggregates [20]. In that research, they have transformed the 3D laser scan data into a 2D image format and utilized technologies developed for digital imaging for assessment of the volume of an aggregate. These research projects demonstrate the applicability of LADAR technology to the assessment of as-built conditions on construction sites.

Embedded sensors are also beginning to be used effectively on construction sites to collect quality-related information. For example, Goodrum et al. completed a pilot study using embedded temperature sensors and data loggers to enable the use of the concrete maturity method to predict concrete strength for cast-in-place components for an industrial facility [21]. This study showed that data collected during inspection could be used to accelerate construction by providing frequent measurements of components as they cure; resulting in lower labor costs 
and a shorter overall schedule. It has also been shown that embedded sensor deployments enable improvement in construction quality control and quality assurance. For example, Inaudi et al. used multiple embedded deformation sensors in a concrete bridge first to inspect strains experienced during the curing process, and secondly to monitor the mean elongation and bending experienced during a six-hour staged relocation process to ensure that the bridge did not experience damage as a result of the move [22]. Embedded sensors are also used in long-term monitoring of such properties as acceleration, vibration, and acoustic emissions well after construction [23]. These research projects and field tests demonstrate the applicability of embedded sensors in varying sized deployments and for time periods varying from days to years

All of these research projects demonstrate that laser scanners and embedded sensors can be applied effectively in a wide variety of construction site conditions and in varying durations of deployment. However, additional development is necessary for preparing for, avoiding, and reacting to technology failures, such as crimped wires and overfilled data loggers. Additionally, while considering frequent inspection of as-built conditions using reality capture technologies, one must consider the upstream and downstream process implications of inspection timing, frequency, level of detail, and personnel commitment, such as modeling time [46]. We have built on these previous research studies in applications of reality capture technologies on construction sites to develop an approach for inspection planning that deploys these sensor technologies and for interpreting the data originating from these sensors for assessment of construction quality.

\section{Overview of the approach}

Figure 1 describes the overall ASDMCon (Advanced Sensor-based Defect Management on Construction Sites) approach and different modules that make it up. The approach consists of modules that enable acquisition and update of the design and schedule information, modules that enable inspection planning and collection of as-built information based on that plan, and modules that analyze the as-built information from a quality control perspective. Some of these modules are commercially available (highlighted as dashed-lines in the figure) and some of them are developed within the research effort.

In terms of commercially available systems, we have used ArchiCAD 8.0 in creating the asplanned product model. For data collection, we have utilized two different types of laser scanners and various types of temperature sensors. The laser scanners that we utilized include a commercially-available Zoller + Fröhlich LARA 25200 (Z+F scanner), and a research test-bed composed of two actuated SICK lasers (CMU scanner). Both generate 3D point clouds as their output. We have conducted experiments with commercially available embeddable sensors such as thermocouples and thermistors produced by intelliRock, Con-Cure, and Microstrain.

As Figure 1 shows, our approach for active project control and management utilizes a core "living" (continuously updated and maintained) project model composed of as-planned, as-built, specification, and defect models. The as-planned model includes product and process information obtained from design and scheduling software systems and represents that information in an integrated manner. Specification modeling enables capturing the construction 
specification information and representing them in a computer-interpretable way within the integrated project model. The as-built model contains product and process information based on the data collected from a construction site. Finally, the defect model contains any product related deviations identified between as-planned and as-built models.

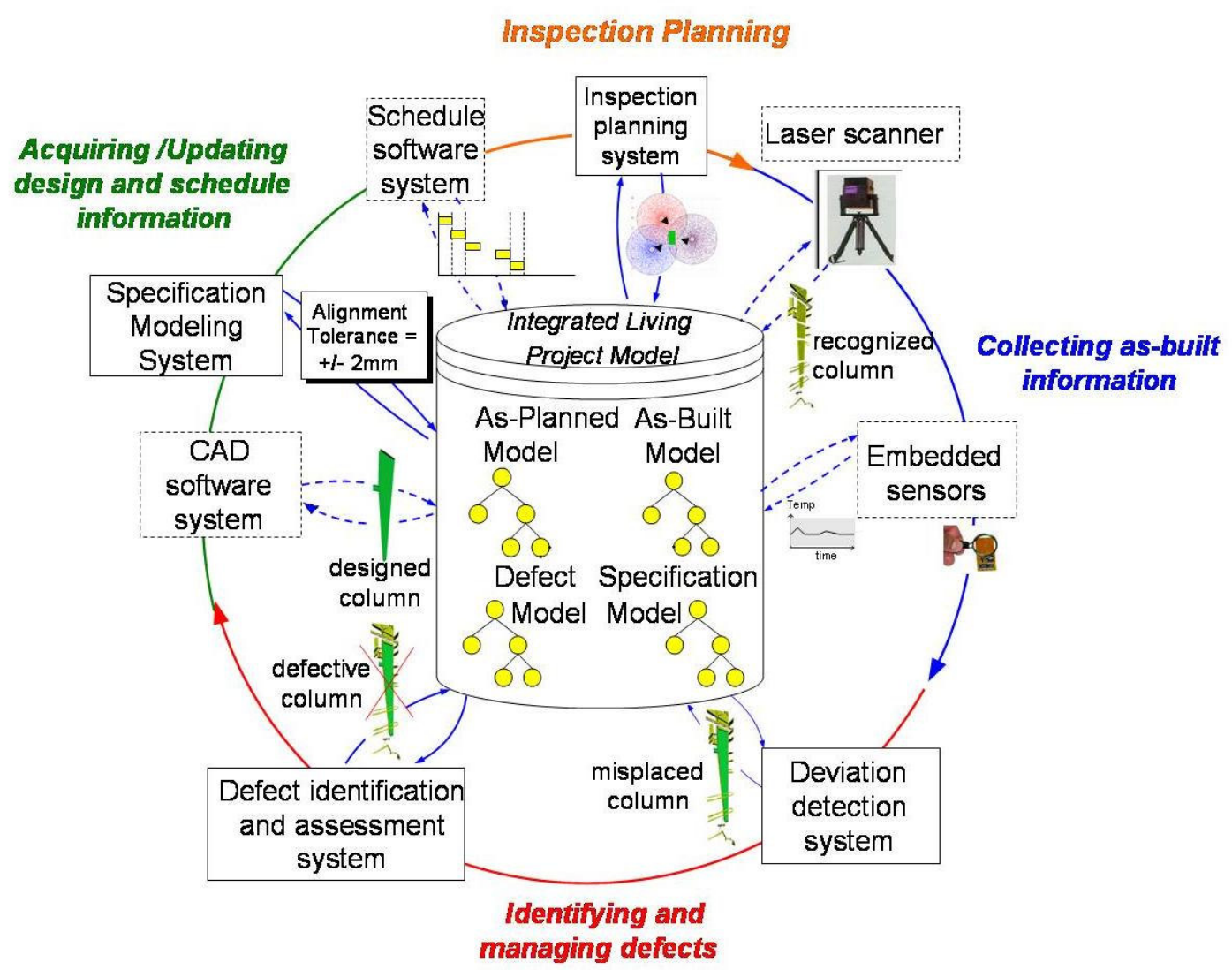

Figure 1. Overall approach for sensor-based quality control

This quality control formalism implemented in this research is composed of five major processes: transforming the design model, identifying inspection goals, planning inspection activities, collecting as-built data, and analyzing data for quality control (Figure 2). The first process transforms a given design and schedule models to create an initial as-planned project model with multiple views necessary to support the subsequent processes. This project model, together with computer-interpretable representations of construction specifications, is utilized to identify inspection goals. Information goals are utilized for inspection planning, during which inspection methods are identified based on the available sensing systems and their properties. With inspection methods selected, sensor locations are optimized and inspection activities are created and added to the project model. This information is used to guide the collection and analysis of as-built data. Analysis of as-built data includes alignment of data collected from multiple scans and recognition of objects within the scanned data. These help in generating an as-built model that can be compared to the as-planned model to identify deviations. Once the deviations are identified, construction specifications are reasoned with to determine if any of the deviations constitutes a defect. At the end of these five processes, a project model that integrates as-planned, as-built, and defect information is generated. Whenever a construction defect exists, 
further actions might need to be taken to correct the defect (in terms of rework) or to incorporate it into the next version of the design. Hence, the overall process outlined in the circle shown in Figure 1 and 2 continues until the completion of a project.

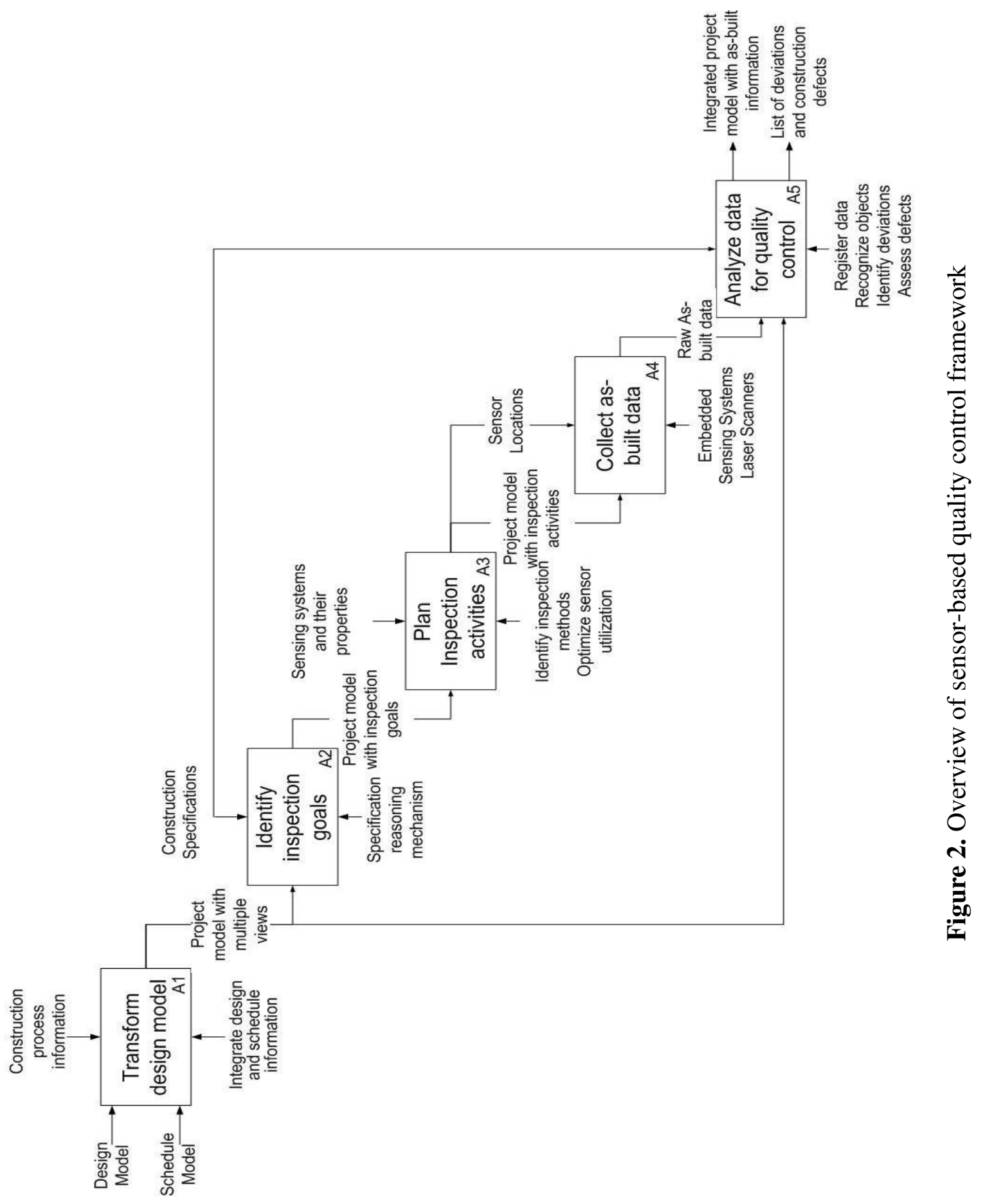

The following sections elaborate further the five processes shown in Figure 2. 


\subsection{Transformation of the design model}

Previous building product modeling research efforts have focused on providing a designcomponent view of a building project. Many researchers [24][25][26] have identified issues of product model progression and elaboration; these become important when the different product models become incorporated into a project model in a construction process. Such project models support the exchange of engineering and other design information with construction information, such as specifications, and support advanced planning and monitoring during construction.

The transformation of a design model into a project model involves decomposition of components. In a previous research, Fischer and Aalami (1996) defined mechanisms that transform a design-centric decomposition of a product model into a production-centric decomposition [27]. Their approach included three sets of transformations; (i) the introduction of temporary structures, (ii) the refinement of components, such as walls, slabs, beams and columns etc., and (iii) the aggregation of components. These three mechanisms were triggered by or closely related to a construction schedule and a set of construction methods utilized. Additionally, the transformation of a design model included other complex relationships with a construction process. Akbas and Fischer (1999) pointed out that the relationships between components can vary in different views over the life cycle of the project [28]. It is clear that whenever there are changes to the product model caused by transformations resulting in new components or new inter-component relationships, the underlying representation needs to be updated.

The mechanisms defined by Fischer et al. (1998) [11], make the reasons for each product model transformation explicit. Therefore, new product models suitable for a particular set of tasks can be generated, and the resulting specific product models can be maintained whenever design and construction methods change. While these previous approaches are suitable and can generate the necessary changes in a product model for the purposes of the research described in this paper, maintaining a product model at a desired perspective and level of detail throughout the design and construction process, without explicitly defined and implemented product model transformation mechanisms, can be cumbersome. Knowing that a variety of engineering tasks require multiple viewpoints, each distinguished by particular interests and emphases, there are multiple representations for certain components in the project model. The transformation mechanisms can be implemented at the database level, with clear specifications from the user. The research described in this paper incorporated such an approach, called sorts [29][30][31], for transforming project model objects as needed by a particular set of engineering tasks, such as inspection planning and deviation detection.

"Sorts" provides an approach to defining representational structures that enable these structures to be compared based on the scope. "Sorts" also presents a uniform approach to manipulating data structures. Individual sorts are class structures identified by compositions of properties. Properties are named entities identified by a type specifying the set of possible values. These can be composed or grouped using one or more constructors. Currently, two constructors are considered: subordinate and disjunctive coordinate compositions of properties. Furthermore, the ability to alter a representational structure by changes to compositional sortal relationships 
facilitates user definition and redefinition of and within the representation. More information on this "sorts" approach can be found in [29][30][31].

\subsection{Identify inspection and measurement goals}

To perform sensor-based quality control, it is critical to know what the inspection goals are, i.e., what components and attributes of components need to be inspected with what accuracy. Determination of inspection goals is a first step towards the selection of appropriate inspection methods and technologies. With the technologies in mind, the appropriate measurement goals, i.e., what measurements need to be made and with what accuracy, can be derived from the inspection goals.

Construction specifications for a project serve as a major knowledge source for identifying inspection goals. These specifications describe the quality requirements for the construction project and, as such, state allowable performance tolerances for the constructed products. This information can be directly translated into inspection goals. However, this is too tedious to be done manually due to the large number of construction specifications that are applicable to a construction project. Therefore, this process needs to be automated as much as possible.

To enable the automation of the inspection goal identification, construction specifications need to be represented in a computer-interpretable form. In this research, we have developed a representation of specifications using contexts, requirements, features, attributes and behaviors to support both automated inspection goal identification and automated construction defect detection [32]. For inspection goal identification, the context of a construction specification can be evaluated to identify a set of components that are possibly related to a given specification. The requirement of a construction specification restricts the applicability of the specification further by imposing additional requirements that are bound to attributes of specific components or behaviors of features. If a specification's context and requirements are satisfied, the attributes and features, together with their corresponding tolerances targeted by a given specification, are determined. These features and attributes of a component and the corresponding tolerances are then represented as inspection goals for the components associated with that given specification.

Knowing only the inspection goals is not sufficient. It is also necessary to know the earliest and latest times that inspections can be performed. With temporal information available based on a given schedule, it is possible to identify windows of opportunity for performing inspections. This temporal information further elaborates the inspection goal description. When determining the windows of opportunity for inspections, one can identify the start dates of construction activities related to a given building component and assign that time as the earliest time that the component can be inspected. In addition, some specifications require inspections of a component to be performed before other components are to be built. In those cases, retrieving the installation times for the succeeding components from the schedule helps in defining the latest time when an inspection can be performed. Thus, windows of opportunity for inspection goals can be determined automatically using the underlying integrated as-planned product and process model, and reasoning about the construction specifications. These windows of opportunity should be determined periodically every time as -planned or as-built is updated and should be linked to the as-built schedule. 


\subsection{Inspection planning}

Given inspection goals, and the desired accuracy and timeframe during which they are to be addressed, one can then select appropriate inspection methods and make decisions about which technologies to use for data collection functions, such as measurement, communication, memory and power, and when and where to use these technologies such that deployment meets inspection requirements effectively. First, it is necessary to identify inspection methods that may be appropriate for a given inspection context. This process, identified in [33], requires determining if an inspection goal is refined sufficiently to be matched to available inspection methods, reasoning with contextual information, such as weather, in order to refine goals further, and heuristically matching goals to methods by reasoning with the attributes to be inspected. Inspection methods are then applied to generate inspection activities and to select inspection technologies such as sensors. Once the inspection technologies are selected, sensor locations can be optimized to ensure adequate coverage of the area to be inspected. The selected set of sensors can be deployed to the optimized sensor locations to make the required measurements. As a result, sensor deployment is integrated into the project models incorporating as-built and asplanned design and schedule.

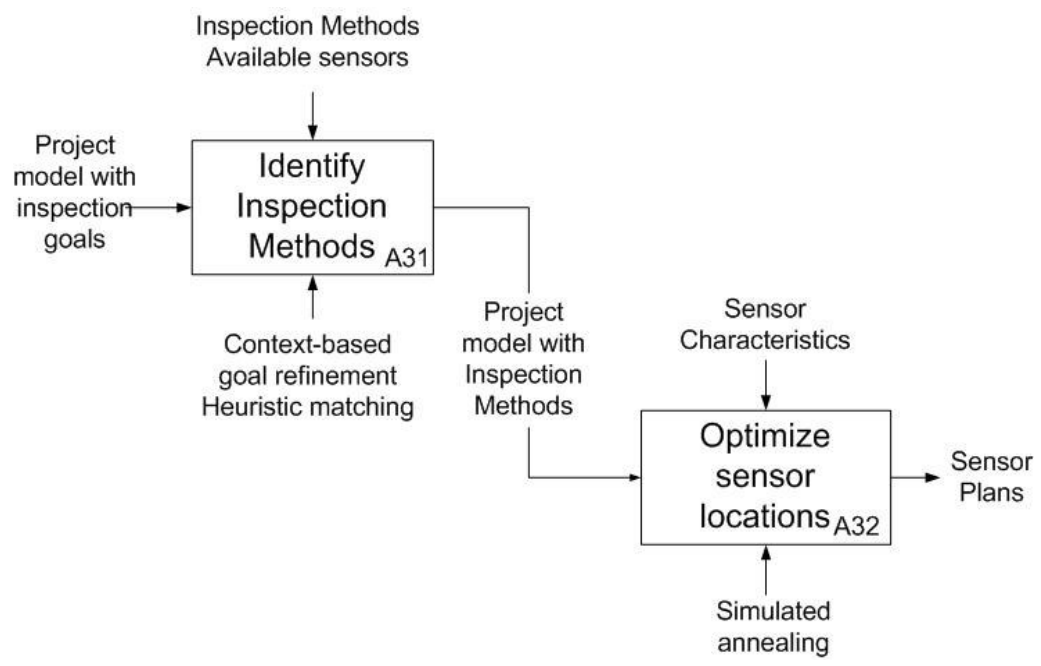

Figure 3. Process model of inspection planning

\subsubsection{Identification of inspection methods}

Goals for inspection may be addressed by multiple types of inspection methods, each requiring different inspection technologies and tasks. In order to make informed decisions about an inspection to be performed, it is necessary to consider the range of possible inspection methods that can be applied in order to satisfy the goals for inspection.

Inspection goals might not be represented at a sufficient level of detail to select appropriate inspection methods, in which case the goals must be refined. This may be because a goal is not defined based on measurable attributes, or because no inspection method is available to address a given inspection goal. Information about the context of inspection can be used to guide how an 
inspection goal should be refined. For example, in a hot-weather context, concrete quality should be evaluated based on durability, compressive strength, workability, and temperature. Given a fully refined goal (i.e. one that can be addressed by an inspection method in a given domain), it is possible to identify various methods capable of addressing the goal, and generate inspection plans. Inspection methods are identified by reasoning with the attributes to be inspected and reasoning with the constraints on how inspections are to be performed, such as constraints on the duration or destructiveness of inspections.

\subsubsection{Optimization of sensor locations}

It is expensive to provide sensor coverage for areas that do not require frequent or even occasional inspections. Instead, it is necessary to optimize sensor configurations for the highest quality data collection, while minimizing cost and time to acquire that data. Three-dimensional laser scanning is a relatively costly process, as it takes time to set up the LIDAR sensor, take a scan, and visually verify the data. In order to minimize the length of interruptions to the construction process during data collection, the fewest number of scans should be used to achieve a desired set of measurement goals. Furthermore, if the necessary data is captured in fewer scans, it may simplify the data analysis process later, as there is less data to process.

Finally, different inspection goals may have different scanning requirements. For example, checking alignment of an anchor bolt may require a high scanning resolution while confirming relative position and orientation of walls and columns requires a scan that captures information over a wide area. Another goal of scan planning is to ensure that scans achieve the required resolution or data density, and that multiple goals will be captured in the same scan if possible. As a result, the user needs to designate which measurement goals must be captured in the same scan in order to fully meet certain inspection goals.

Inputs to the scan planning process are the as-designed model for the current stage of construction, the scanner specifications (based on the scanner selected during inspection planning), and the corresponding measurement goals. Outputs are an optimal set of sensing locations and scan angles to achieve the given goals, and a motion plan (path) to travel between sensing locations.

The first step in scan planning is to group construction measurement goals into clusters, simplifying the planning space in order to reduce computational complexity. For each cluster, the planner generates the space of potential sensor placements for a set of information goals and selects a minimal set of subspaces to take advantage of views that can achieve multiple goals simultaneously. Sensing locations are chosen that maximize the resolution achieved for each goal, and a path is generated to minimize the transit cost between the various sensing locations within each cluster. Finally, a path that minimizes the transit cost between sensing locations is generated. At various points in the process, relevant data is saved so that it can be reused should the plan need to be updated. Further details on scan planning are described in [34]. 


\subsection{Data collection}

Based on the inspection methods identified and the optimized scanner locations, one can start collecting data on a construction site. In this research, data collection efforts included two different types of scanners to collect the geometry information, and three different types of temperature sensors to assess the strength of a cast-in-place concrete in various construction job sites.

We have experimented with commercially-available Zoller + Fröhlich LARA 25200 (Z+F scanner), and an internally developed scanner (CMU scanner). Both scanners generate 3D point clouds as their output. The $\mathrm{Z}+\mathrm{F}$ scanner has a $360 \times 70$ degree field of view and a 25.2 meter maximum effective range. It has a data rate of 120,000 samples per second, acquiring an $8000 \mathrm{x}$ 1400-pixel scan in ninety-three seconds. Each point in the scan contains a fifteen bit range measurement and a sixteen bit reflectance value. The noise on the range measurements is zero mean Gaussian with a standard deviation of $2.67 \mathrm{~mm}$ for a $20 \%$ reflective surface at thirteen meters. The $\mathrm{Z}+\mathrm{F}$ scanner has the advantage of delivering $3 \mathrm{D}$ data at very high resolution in a reasonable amount of time (Figure 4a). For example, at a range of ten meters, a surface will be sampled on a grid with 9mm spacing. The CMU scanner consists of a SICK (LMS 291) laser line scanner mounted on a panning mechanical actuator. It has a 130x100 degree field of view and acquires 800x400 pixel range image in approximately 10 seconds. At ten meters, a surface will be sampled on a grid with $4 \mathrm{~cm}$ spacing- about a fourth of the spatial resolution of the $\mathrm{Z}+\mathrm{F}$. The noise in the CMU scanner's range measurements has a standard deviation of $6 \mathrm{~mm}$ for a surface of thirteen meters. The maximum range of this scanner is eighty meter. The CMU scanner is much faster than the $\mathrm{Z}+\mathrm{F}$ scanner in acquiring as-built conditions and provides sparse scanning as a possible way of collecting the data (Figure 4b). Figure 4 shows the scanned data of a column footing obtained from the two scanners.

It is important to understand how sensor resolution and range accuracy translate into the ability to detect construction defects. For example, can the $\mathrm{Z}+\mathrm{F}$ scanner detect a column that is out of position by $6 \mathrm{~mm}$ ? The answer depends on a number of additional factors beyond the underlying sensor accuracy, including measurement goal type, object size, surface orientation, and even surface material. Some measurement goals, such as determining the location of anchor bolts, require point measurements, while other goals, such as determining the plumbness of a column, involve measurements on relatively large surfaces. The accuracy of area-based measurements can be much higher than that of the individual sensor measurements because data fitting methods, such as plane fitting, will average out the single measurement noise, provided that it is unbiased. Object size also affects the accuracy of area measurements. All other things being equal, the plumbness of a six meter column can be measured more accurately than that of a three meter column because twice as much surface area can be used for the plane fitting. Surface orientation affects measurement accuracy due to reduced resolution of obliquely viewed surfaces. For example, the side of a column viewed from a sixty degree angle will have a resolution half as dense as the front of the same column viewed straight on. Finally, surface material can affect measurement accuracy. Dark-colored surfaces absorb laser light, resulting in noisier range measurements. At the other extreme, highly reflective surfaces, such as polished metal, can act as a mirror, resulting in an incorrect range measurement or no measurement at all. 


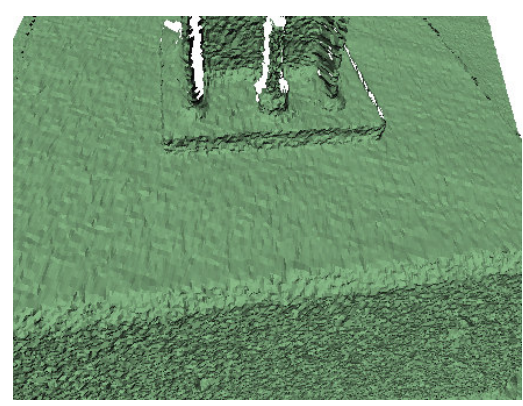

Figure 4a. As-built data obtained from the Z+F scanner

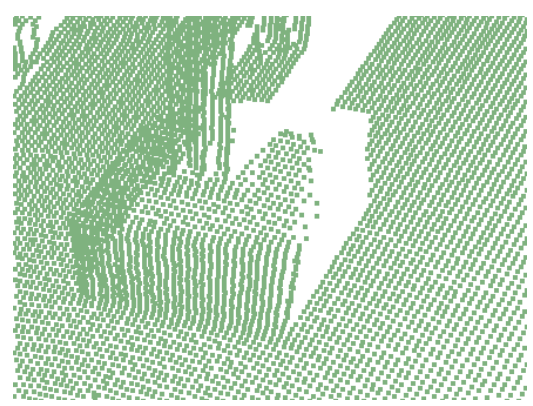

Figure 4b. As-built data obtained from the CMU scanner

Figure 4. Data acquired about a column footing from two different scanners

As can be seen from Figure 4, the data collection process and the resolution of the data collected can be quite different based on the scanner utilized. The quantity of the measurement goals for a given scanning session and the detail and accuracy required for each measurement goal determine the choice of scanner. For example, if one needs to have detailed data about anchor bolts, a higher resolution scanner placed close to the location of objects of interest might be needed. On the other hand, if one needs to know the locations of a large number of columns in a row, a scanner with lower resolution and higher range located further away from the component might suffice. These experiments further demonstrate the need for formally identifying the measurement goals, the corresponding inspection methods (which include the choice of technology to utilize), and for optimizing scan locations prior to acquiring data on sites. Other lessons learned associated with utilization of the two laser scanners on construction sites include the difficulties of moving the equipment over rocks, mud, and temporarily stored materials on the site; the need for power to run this equipment; and the impact of the extreme temperatures on the performance of the sensors [35].

We have also experimented with various commercially available embedded temperature sensing systems. We selected systems that differ from each other in terms of whether communication, computation, measurement, and data storage functions were performed in-situ, in-situ/mobile, or ex-situ. Initial experiments showed that requirements of supporting sensing system technologies, such as the amount of data storage, must be designed to support an inspection task as well as to accommodate the uncertainty in timing of construction operations [35]. Further experiments with embedded sensing have focused on identifying distinguishing factors in decisions related to selection and use of sensing technologies.

\subsection{Data analysis for quality control}

The as-built data collected from construction sites using sensor systems needs to be further processed to enable automated analysis for quality control. Figure 5 shows a process model of the as-built data analysis process for automated quality control. Laser scanners provide point clouds in local coordinate for each scan location. To have a complete understanding and assessment of as-built conditions on a construction site, the individual scans need to be aligned 
with one another - a process called registration. The registered data provides a more comprehensive 3D model of the site. However, it is still in a point-cloud form. Object recognition provides a way to identify objects from a scene and reverse engineer the objects identified to highlight and represent the as-built conditions of the building components within the as-planned model in an object-oriented way. At the end of object recognition, an object-oriented representation of as-built conditions is integrated into the project model. Once an integrated asplanned and as-built model is created, the data from both models can be compared to identify deviations and to assess whether any of the deviations identified constitutes a construction defect.

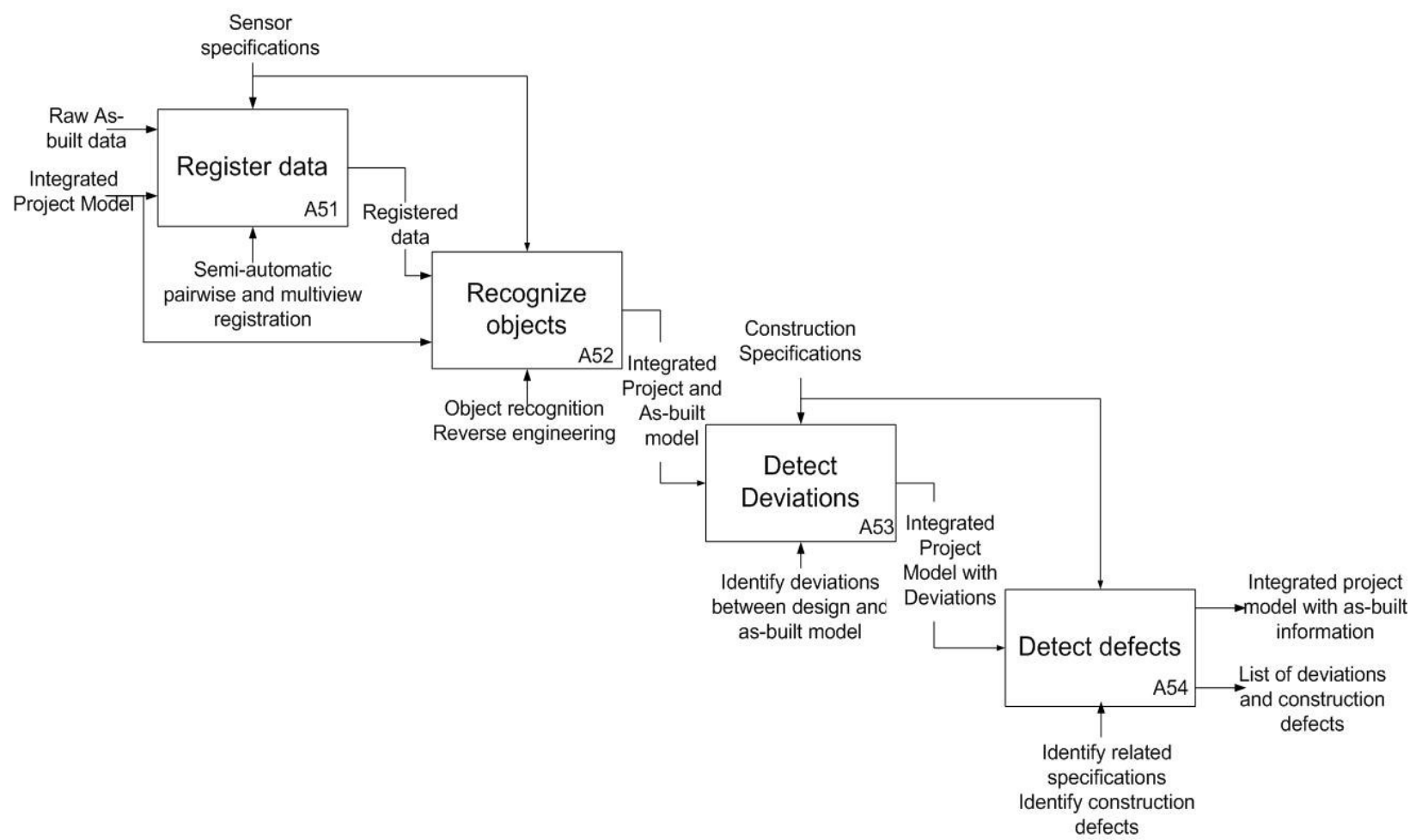

Figure 5. Process model of data analysis for quality control

\subsubsection{Registration}

The as-built data obtained from the laser scanners are in the form of 3D point sets known as point clouds. The point cloud from each scan is represented in the local coordinate system of the scanner, which makes it impossible to perform direct comparisons with the as-designed model for defect detection and analysis. Therefore, it is necessary to align all of the scans in a common coordinate system - a process known as registration. Furthermore, the collectively registered asbuilt scans must be registered with the as-designed model to enable reverse engineering and deviation detection.

There are a number of established methods for registering 3D point clouds. First, the sensor can be augmented with a pose estimation system that records the scanner's position and orientation for each scan [36]. Second, the environment can be augmented with markers, known as 
fiducials, with known locations, which are then detected in the 3D data and used to aid in the registration [37]. Third, the scans can be manually registered, for example, by specifying two or three pairs of corresponding points in two scans [38]. Finally, automated methods based on computer-vision algorithms can be used to automatically register views without any knowledge of the scanner position or orientation [39].

Currently we use the manual registration method, with the goal of migrating to the automatic registration method in the near future. Our current automatic registration algorithms require approximately fifty percent overlapping data to succeed. The next generation of the algorithm will be extended to handle a lower amount of overlap that is dictated by the need to minimize the total number of scans collected.

Once the registration of pairs of scans is accomplished, a second registration phase involving all of the scans is necessary. The process of sequentially registering a series of data sets leads to accumulation of error, and the resulting model will not be geometrically accurate. This error can be eliminated by simultaneously registering all the scans, a process known as multi-view registration. Several multi-view registration algorithms have been proposed [40][41][42]. Neugebauer's algorithm [41] has proven effective in our work.

Finally, the as-built model must be registered with the as-designed model. This problem is identical to the pair-wise registration problem described above, and therefore, the same methods are applied.

\subsubsection{Object recognition}

Once the as-built model is constructed and registered with the as-designed model, it is possible to perform quality control analysis. However, since the as-built model is in the form of a point cloud, this analysis must necessarily take place at the lowest level (i.e., raw point data). We hypothesize that more effective analysis can be accomplished by further processing the as-built data to enable comparisons at a higher level. Object recognition serves this purpose by providing a bridge from the low-level point data to a high-level component level. For example, given a 3D model of a column, that column can be detected in the as-built data, and its position determined through the use of object recognition.

Previous research in 3D computer vision has demonstrated the ability to detect and localize freeform objects within 3D scenes [43][44][45]. The key to this technology is the use of local shape descriptors, which encapsulate the surface shape of parts of a 3D object model to be recognized (the query model). The same shape descriptors are then computed at points in the as-built model (the scene). Descriptors for points on the target object in the scene will be similar to those computed for the query model. Once the object is recognized, the matching descriptors can be used to determine the pose of the query model in the scene.

This basic object recognition approach has been applied to rigid objects, and more recently to articulated objects. Currently, we are working on symmetry analysis as well as extensions to 
enable non-rigid objects to be recognized. In particular, we are seeking ways to allow recognition of parametrically defined query models, such as a column with variable height.

\subsubsection{Deviation and defect detection}

Identifying spatial and material quality related deviations is the first step in discovering construction defects. Identification of deviations can be accomplished by comparing the processed as-built data obtained from sensors to the given design model. Once a deviation is found, it can be evaluated based on related construction specifications to determine whether the deviation identified violates any specification and thus constitutes a construction defect. If this is the case, corrective actions need to be planned and implemented to resolve the defect. Identification of deviations is also important in preventing the occurrences of defects. If a deviation between the design and the as-built conditions is known, this knowledge can be incorporated in the planning of succeeding construction activities to avoid propagation and/or amplification of the deviations which could result in defects [6].

The research project described in this paper incorporates a model-based deviation detection approach which assumes that all as-built and design information is represented in the integrated project model. The as-built information integrated in the model thereby is extracted and derived from the raw-data received through the deployed reality-capturing technologies. The raw-data is also linked to the integrated project model, so that no loss in data or resolution occurs. Within this model, the approach compares the design information to the related as-built information to identify deviations. A simple comparison of the values of the attributes of the objects within the as-built model to the objects within the design model is not feasible since the representation of the design information and the representation of the as-built information are likely to differ in their structures. The differences can stem from different levels of detail of the data, different levels of hierarchical decompositions, and differences in the actual representation of the same information resulting from the flexibility of the project model used [32]. Thus, a one-to-one comparison of design and as-built information is not appropriate.

Instead, a feature-ontology and attribute-ontology based deviation detection approach is needed. Ontology for features and attributes developed from construction specifications enables the development of a deviation identification mechanism that is not dependent on the structure of the information representation within the integrated project model. In this case, the deviation detection mechanism searches for deviations in the features and attributes. However, additional mechanisms need to be implemented to extract the relevant feature/attribute from the underlying information representation in a given integrated project model. Due to the possible repetition in the structure of the information representation in the integrated project model, many of the feature extraction mechanisms are reusable for different objects in the model. If raw as-built data is available and linked to the integrated project model, it is used for comparison to the design information extracted from the integrated project model to avoid any loss of information resolution. Any deviations found will then be mapped to the respective features and/or attributes defined in the ontology. For example, deviation detection based on scanned data will compare the raw point clouds with the design information. The deviations found are then mapped to the specific features or attributes, e.g. surface-deviations or location-deviations, of the affected component. 
Since the deviation detection will be performed frequently throughout the construction process, it is expected that the as-built part of the integrated model will change over time and will achieve its highest level of completeness only when construction is finished. Thus, comparing design information to as-built information will not always be possible, since as-built information can be expected to be missing. Therefore, the deviation detection mechanism needs to reason about any scheduling information available in the design part of the integrated project model to identify whether a component is expected to be built at the time of the deviation detection or not. If a component is scheduled to be built at some later point after the time of the deviation detection, the as-built information of the component can be expected to be missing. Thus, the component does not need to be checked for deviations yet. It might also happen that the as-built information of a component is not available in the integrated project model, even though the component is expected to be installed. In that case, the system will point out to the user that the information is missing and ask the user to provide the missing as-built information or to update the schedule to reflect the actual delay. In certain cases, parts of the design information might be missing since the design might not be detailed enough, for example, to graphically show the geometric representation of an anchor bolt. . If such information is needed for the deviation detection, the user needs to intervene to update the integrated project model by providing more detail on the design information. The user can choose to provide the missing information or, if he/she finds that providing the information is too tedious, continue without providing the information. He/she will then be notified later about which checks could not be performed and which specifications could not be tested due to the missing information.

The deviations found need to be further processed in relation to the construction specifications to assess whether a deviation is a defect. The evaluation of deviations is done by comparing them to the targeted quality standards, expressed in construction specifications, for the related components. This process utilizes the construction specification model developed for generating inspection goals and compares the amount of deviations to the corresponding allowable tolerances defined in specifications. If a given deviation exceeds the allowable tolerance, then it constitutes a construction defect and thus further actions need to be taken to correct the defect or to incorporate it into the next version of the design. Both of these actions impact the as-planned model either with addition of rework activities in a given schedule or modifications in the design model.

\section{Validation}

We have performed case studies at four different construction job sites: (1) a 1,000 square foot footbridge, (2) a 36,000 square foot one story warehouse construction (Figure 6), (3) a 50,000 square foot one story office and production facility (Figure 7), and (4) a 133,000 gross square foot nine-story commercial construction project with five floors for office space above a fourlevel parking garage (Figure 8).

The comparison process between as-designed and as-built model requires that the as-designed model be presented at a comparable level of detail to the laser scanned as-built model. In order to compare with the geometric features, the as-designed model needs to be three-dimensional and 
highly detailed. We created 3D design models of the projects prior to the start of the data collection and data analysis processes. Figure 7a shows an example of a 3D model created for the Case Study site \#3. These 3D models were very detailed in nature due to the fact that some construction specifications were targeting detailed objects and their locations. Figure 7c shows the initial 3D model created for a column and $7 \mathrm{~d}$ shows the corresponding as-built data collected form the site. We periodically hired undergraduate students to create these 3D models. For case study \#2 (Figure 7), one undergraduate student spent about twenty hours a week for one semester to generate a highly detailed as-designed model, including steel frames, x-bracing, corrugated siding, etc. In this case study, the available design information were 90\%-complete design documents depicted in $2 \mathrm{D}$ blue prints. Starting with a CAD model would significantly reduce the generation time for 3D models.

Once the 3D design model for each project was created, we started planning for the inspection and data collection operations and visited the job site periodically to collect the necessary data. Depending on the construction activity and the corresponding data collection needs, we averaged about one to two weeks between data collection visits. Each data collection visit produced twenty to seventy scans. Each scan took approximately one to two minutes, and each relocation between scan locations took approximately three minutes. Embedded sensing data collection duration ranged from hours up to one month. Further detail on case study experiences and technological and process assessment of using these for frequent as-built assessment are documented in [35] and [46].

Figures 6, 7 and 8 show examples of the data collected from different construction sites. While Figure 6a shows that an uncluttered site can be measured with one $360^{\circ}$ scan from one location, Figure $7 \mathrm{c}$ shows that a moderately cluttered site can be measured in approximately thirty $130^{\circ}$ scans from twenty locations. Figures $6 \mathrm{~b}, 7 \mathrm{~d}$ and $8 \mathrm{~b}$ show up-close views of the data collected by focusing on data collected on individual columns at a given job site and assessing whether the data captured from laser scanners could be easily associated with the lower level objects modelled in the detailed 3D design models. The images in Figure 6 and 7 show the intensity data generated and collected using the higher resolution $\mathrm{Z}+\mathrm{F}$ scanner, while the images in Figure 8 show point cloud data collected by the lower resolution CMU scanner. Our assessment shows that while higher resolution scanners are needed for identifying and reasoning about higher levels of design information represented in the 3D design model, such as anchor bolts, to get a quick assessment about the locations and the shapes of major components, such as columns and beams, the lower resolution scanners would suffice. 


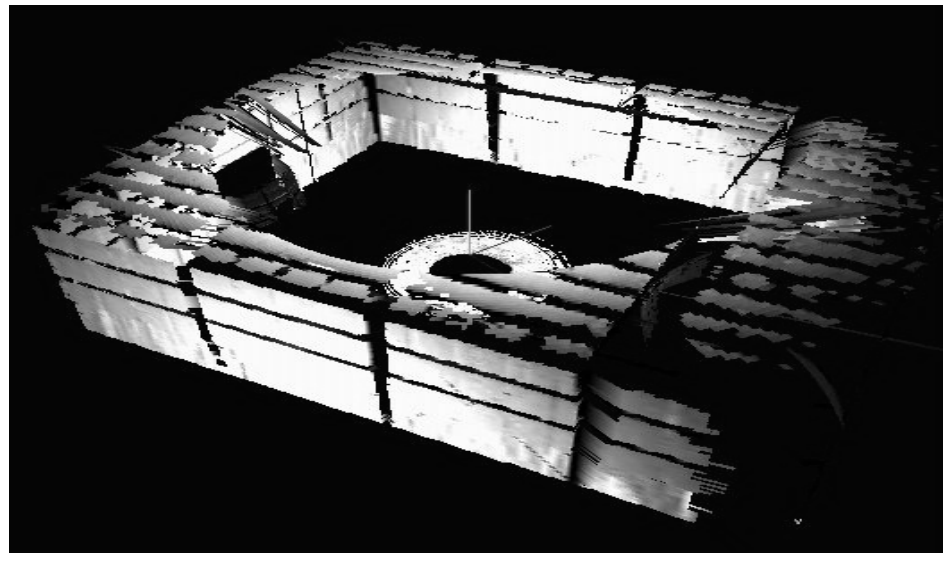

Figure 6a.

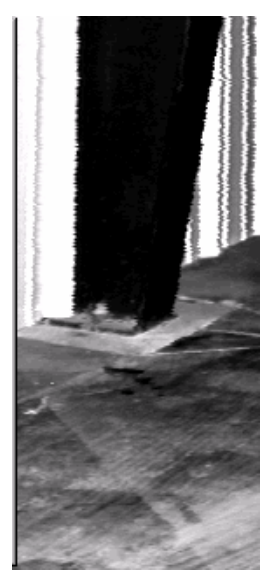

Figure 6b.

Figures 6a and 6b. Scanned data from the Case Study \# 2.

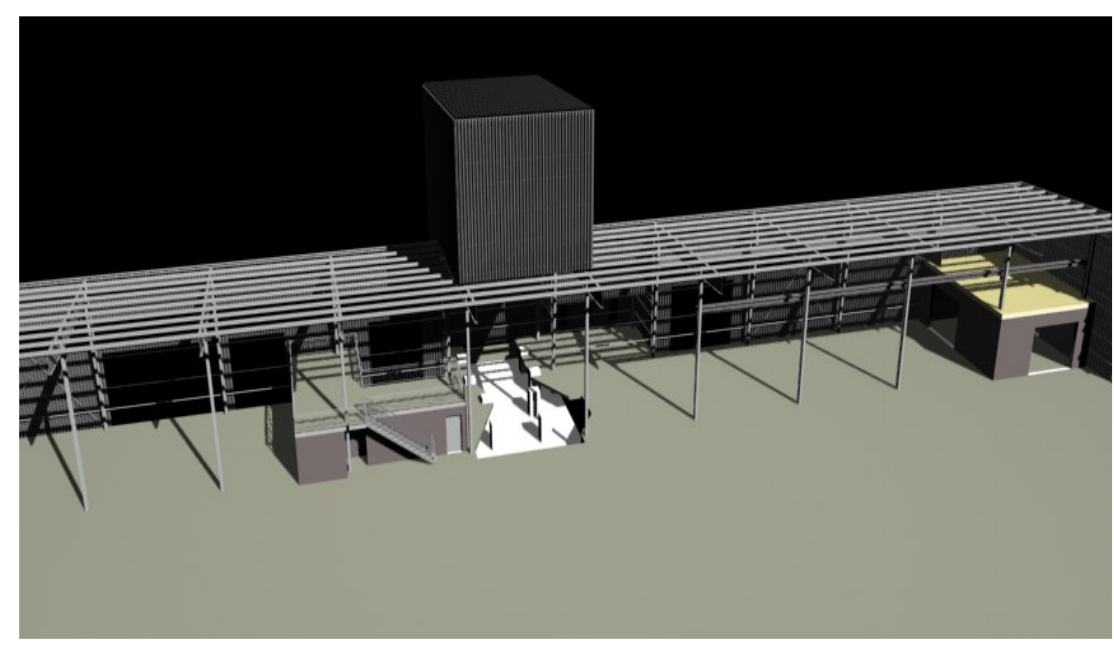

Figure 7a. Initial highly detailed 3D design model of the site

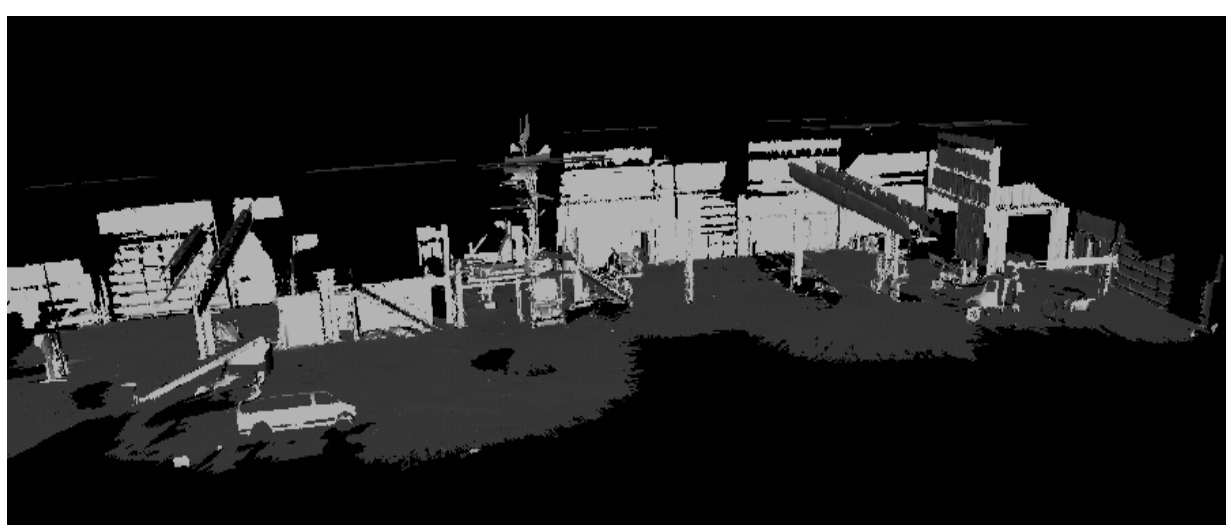

Figure 7c. 3D as-built data collected from the site

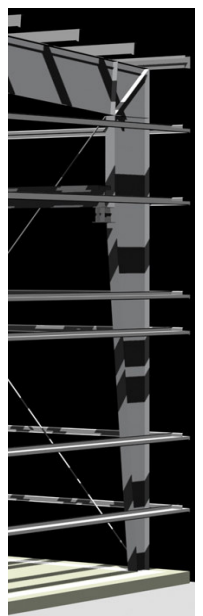

Figure 7b. 3D design model of a column

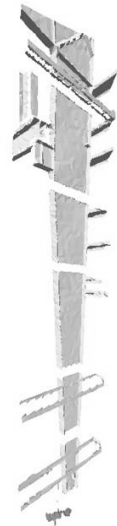

Figure 7d. As-built of the column shown in $7 \mathrm{~b}$

Figure 7. 3D design model and as-built data collected during Case Study \#3 


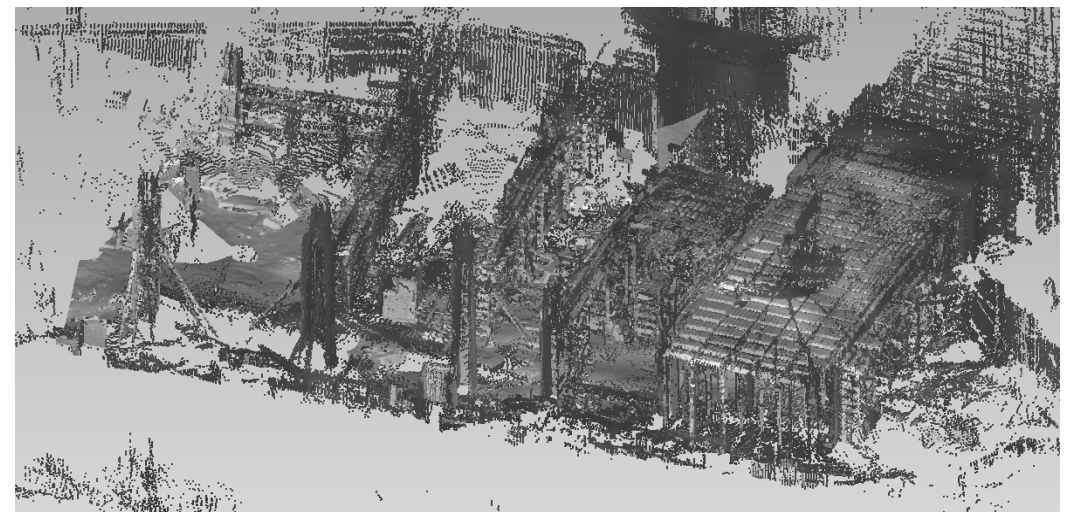

Figure 8a.

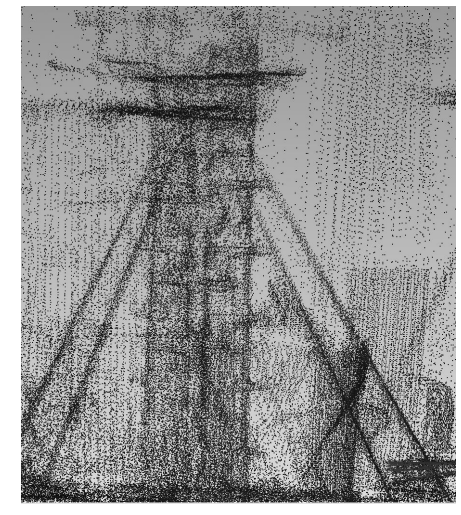

Figure $8 b$.

Figure 8a and 8b. Scanned data from Case Study \#4.

The three building project case studies vary in terms of their sizes and types; providing a test bed to evaluate the generality of the overall formalism. Site conditions were also quite different in all three job sites. The first two job sites (Figures 6 and 7) were moderately congested and there were fewer temporary structures and less material stored on the site. The third job site (Figure 8) was highly congested due to the fact that the site footprint was small and was tightly bounded on all four sides. As a result, a significant amount of material was stored on the site. In addition, since most of the work was cast-in-place, the last job site included a significant number of temporary structures, such as shoring and formwork to be stored and staged at the site. These observations further emphasize the need and the approach for developing formal inspection and scan plans prior to any data collection process.

The designs of the first two building sites were very different than the third site. The first two were mostly steel structures including a large number of components that were small in size, such as anchor bolts, $\mathrm{x}$-braces, and girts, and the construction specifications required reasoning about the location of these small components. The third project consisted of mostly cast-in-place concrete components with challenges associated with the reasoning about the locations and shapes of temporary structures and rebar.

The data collection efforts mostly occurred during the September-March time frame, with highly different temperature ranges, varying from $-7^{\circ} \mathrm{C}$ to $30^{\circ} \mathrm{C}$, and the resulting surface conditions of the site varying from a finished surface to muddy, icy, and uneven surface. These further challenged the data collection and data analysis processes as described in [35] and [46].

Our higher level formalism for automated quality control worked well in all of these highly different cases with varying complexities. This suggests some generality of our approach for the structural and enclosure elements within the commercial building construction domain. In certain cases, our approach has lead to more efficient data collection and processing. For example, during a data collection effort in the second case, the site had twenty-three information goals and two hundred forty-six obstacle vertices. Given a four to five minute estimate to scan, it would take approximately one and a half hours to scan each goal once. Sensor planning took 
forty-three seconds to generate a sensing plan with seven sensing locations. Scanning from the sensor plan would take approximately thirty minutes [34].

\section{Example of deviations identified in the case studies:}

In these case studies, we have identified several types of deviations. An example of a coarse level deviation identified was related to a design change that was not captured in the design documents. Figure 9a shows the design model of a portion of a manufacturing plant. The original design shows three windows and an opening for a loading deck. Figure $9 \mathrm{~b}$ show the original design and the as-built data collected from laser scanners in an integrated way. As the as-built model shows, none of the window or loading deck openings highlighted in the original design exist in the final conditions at the site.

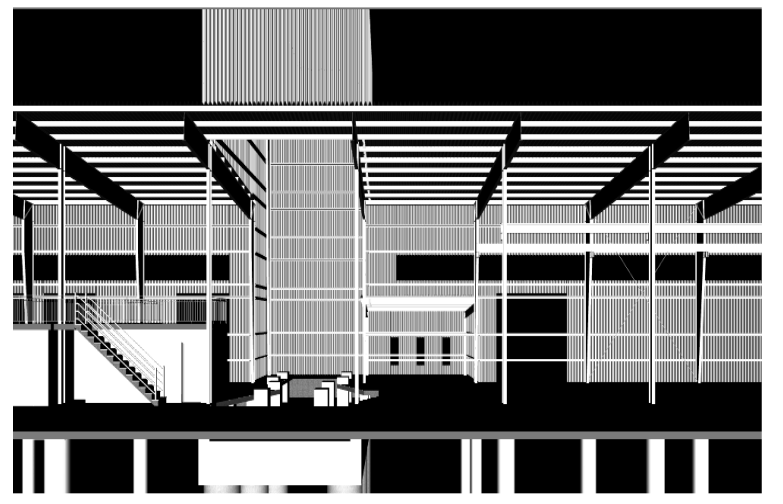

Figure 9a. Original design model of a portion of the site

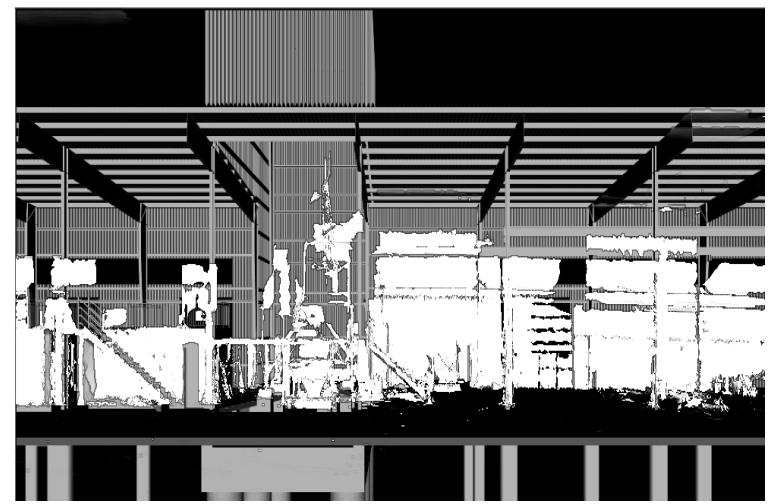

Figure 9b. Integrated view of design and as-built models with deviations highlighted

Figure 9. Examples of design change related deviations identified in Case Study \#2

In addition to obvious coarse level deviations, we have also successfully identified many small scale deviations that would be harder to identify without using the formalism described in this paper, and yet would have important implications about the performance of a facility. An example of such a deviation is changes in the surface and thicknesses of a wall or a column as shown in Figure 10. Such deviations not only have aesthetic issues, but also possible structural implications, such as not being able to provide minimum concrete coverage of reinforcing steel specified by specifications like ACI 318 [47]. When creating a cast-in-place concrete wall, forces being applied to the wall's formwork during concrete-placement can lead to movements and deflection in the formwork. Among others, ACI 347 [48] identifies "failure to inspect formwork during and after concrete placement to detect abnormal deflections or other signs of imminent failure that could be corrected", "failure to construct formwork in accordance with the form drawings", and "lack of proper field inspection by qualified persons to ensure that form design has been properly interpreted by form builders" as common construction deficiencies. Thus, such deviations in the formwork can go undetected. Also, when the formwork or the final product is inspected, usually only sparse measurements are performed, i.e., only a few points on the formwork or the final product are checked. When using laser-scanning technology, a more comprehensive check can be performed, because more points are measured. The resulting 3D asbuilt model can be overlaid with the 3D-design model and deviations within the wall's surface 
can be shown, rather than only deviations at certain points. This allows for a more comprehensive identification of deviations. Additional tests, like checking the actual concrete coverage in areas with alarming deviations, can be performed if needed and, if necessary, immediate measures to counteract certain deviations or to resolve problems can be introduced. Figure 10 shows such surface deviations identified on cast-in-place concrete elements in case study \#2.

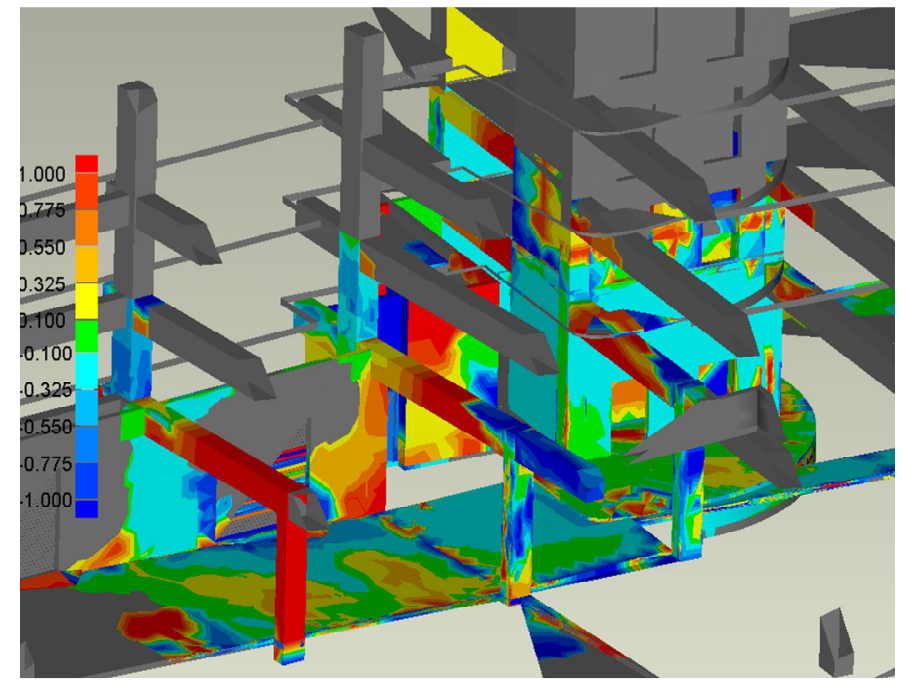

Figure 10. Surface deviations identified through overlaying design and as-built models

In addition to the deviations identified using laser scanners, we have also identified some deviations using embedded sensors. In the small foot bridge project, we used three types of embedded temperature sensors for evaluation. The selected sensors varied according to how data was collected: wirelessly, wired with integrated data logger, and wired with external data logger. The details of the embedded sensors utilized and the technological performances and assessments of those sensors are provided in [46]. During this case study, we identified that a different concrete was used on a grade beam than was specified by monitoring its temperature over time and comparing that to the temperature-time factor developed for a sample of the expected concrete. Figure 11 shows the strength predicted by applying the maturity method with the use of data collected from temperature sensors embedded within concrete components. 


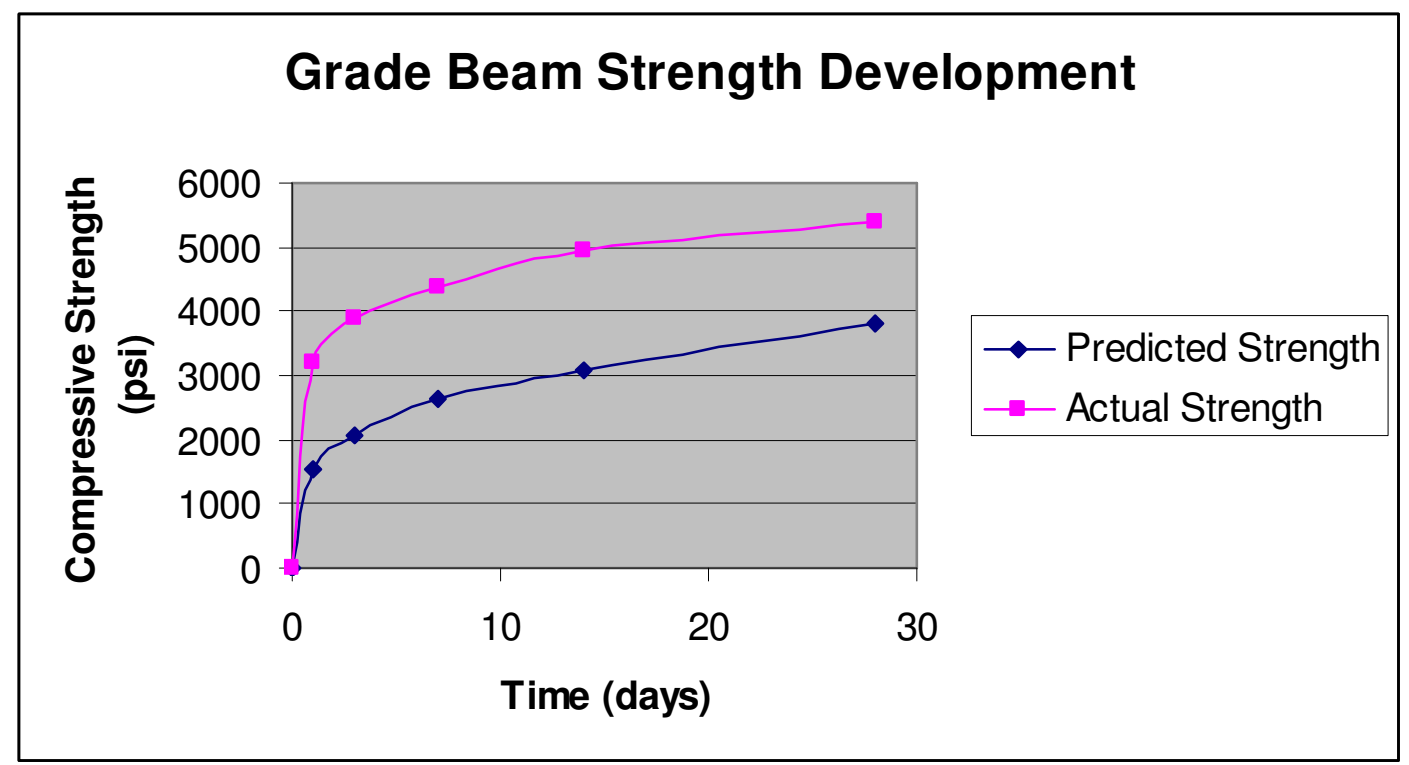

Figure 11. An example of a design change related deviation identified in Case Study \#1 using embedded temperature sensors

\section{Conclusions and future work}

Reality capture technologies have matured sufficiently to be utilized for as-built data collection on construction sites. These technologies can be leveraged to improve project quality control processes. A formalism is needed to utilize these technologies effectively and to analyze the data collected from these instruments to enable active quality control. Our formalism for using advanced sensor systems and integrated project models for active quality control includes five basic process steps: creating as-planned project model information, identifying inspection goals, inspection planning, data collection, and defect detection and management. Our utilization of the formalism on four construction sites suggests that the generality of the overall approach and its possible technical effectiveness in data collection and deviation detection. Future research includes further refining the algorithms implemented in support of this formalism and incorporation of other reality capture technologies to the automated quality control framework. In addition, we hope to perform detailed studies on assessing the cost effectiveness of the formalism described in this paper.

\section{Acknowledgement}

The project is funded by a grant from the National Science Foundation, CMS \#0121549. NSF's support is gratefully acknowledged. Any opinions, findings, conclusions or recommendations presented in this paper are those of authors and do not necessarily reflect the views of the National Science Foundation. Authors also acknowledge the other faculty working on this research project: Jim Garrett, Martial Hebert, Ramesh Krishnamurti and Scott Thayer. 


\section{References}

[1] J. L. J. Burati, J. J. Farrington, Cost of quality deviations in design and construction, Source Document 29, The Construction Industry Institute, The University of Texas at Austin, Austin, Texas, 1987.

[2] P.E. Josephson, Y. Hammarlund, The causes and costs of defects in construction: A study of seven building projects, Automation in Construction, vol. 8 (9), Elsevier, New York, USA, 1999, pp. 681-687.

[3] L. Patterson, W. Ledbetter, The cost of quality: A management tool, Excellence in the Constructed Project, Proceedings of Construction Congress I, San Francisco, California, ASCE, 1989.

[4] N. D. Opfer, Construction defect education in construction management. ASC proceedings of the 35th Annual Conference, California Polytechnic University, ASC, 1999.

[5] J. Yates, E. Lockley, Documenting and analyzing construction failures. Journal of Construction Engineering and Management, vol. 128 (1), ASCE, New York, USA, 2002, pp. 8-17.

[6] Cyra Technologies, Inc., Case Studies, http://www.cyra.com/case_studies/case_studies.html, (accessed June 2004)

[7] B. Foltz, 3D laser scanner provides benefits for PennDOT bridge and rockface surveys, Professional Surveyor, vol. 20 (5) GITC, Frederick, MD, USA, May 2000, pp. 22-28.

[8] M. Hashemi, D. Reinhart, A new approach in plant physical documentation and information management for existing facilities using laser scanning, imaging, photogrammetry, Intelligent Processing and Manufacturing Materials, Vancouver, British Columbia, Canada, July 29 - August 3, 2001.

[9] P.J. Tikalsky, D.G. Tepke, Concrete maturity progress: a survey of departments of transportation, Transportation Research Record of the Transportation Research Board, Paper No. 01-2939, Washington, D.C., January 2001.

[10] B. -C. Björk, A unified approach for modeling construction information, Building and Environment, vol. 27 (2), Elsevier, New York, USA, 1991, pp. 173-194.

[11] M. Fischer, F. Aalami, R. Akbas, Formalizing product model transformations: case examples and applications, Artificial Intelligence in Structural Engineering: Information Technology for Design, Collaboration, Maintenance, and Monitoring, Lecture Notes in Artificial Intelligence 1454, I. Smith (Ed.), Springer, Germany, 1998, pp. 113-32.

[12] T. Froese, M. Fischer, F. Grobler, J. Ritzenthaler, K. Yu, B. Akinci, R. Akbas, B. Koo, A. Barron, J. Kunz, Industry Foundation Classes for Project Management - A Trial Implementation, Electronic Journal of Information Technology in Construction, vol. 4, ITCon, 1999, pp. 17-36.

[13] K. Yu, T. Froese, F. Grobler, International alliance for interoperability: Industry foundation classes, Computing in Civil Engineering: Proceedings of the International Computing Congress, ASCE, Boston, MA, October 19-21, 1998, pp. 395-406.

[14] B. Akinci, J. Garrett, M. Patton, A vision for active project control using advanced sensors and integrated project models, Specialty Conference on Fully Integrated and Automated Project Processes, Virginia Tech, Blacksburg, VA, ASCE, January 23-25, 2002, pp. 386397. 
[15] M. J. Riley, C. J. D. Pickering, Automated total quality management for the construction industry, Computing in Civil and Building Engineering, ICCCBE-V, Anaheim, CA, ASCE, 1993, pp. 1539-1546.

[16] M. Battikha, QUALICON: Computer-based system for construction quality management, Journal of Construction Engineering and Management, vol. 128 (2) ASCE, New York, USA, 2002, pp. 164-173.

[17] S. Chin, K. Kim, Y. Kim, A Process-Based Quality Management Information Systems, Automation in Construction, vol. 13 (2), Elsevier, New York, USA, 2004, pp. 241-259.

[18] G. Cheok, W. Stone, R. Lipman, C. Witzgall, Ladars for construction assessment and update, Automation in Construction, vol. 9 (5-6), Elsevier, New York, USA, 2000, pp. 463477.

[19] S. Kwon, F. Bosche, C. Kim, C. Haas, K. Liapi, Fitting range data to primitives for rapid local 3D modeling using sparse range point clouds, Automation in Construction, vol. 13(1), Elsevier, New York, USA, 2004, pp. 67-81.

[20] H. Kim, C. Haas, A. Rauch, C. Browne, Dimensional ratios for stone aggregates for threedimensional laser scans, Journal of Computing in Civil Engineering vol. 16 (3), ASCE, New York, USA, 2002, pp. 175-183.

[21] P.M. Goodrum, J. Dai, C.R. Wood, M. King, The use of the concrete maturity method in the construction of industrial facilities: A case study, FIATECH Report January 29, 2004.

[22] D. Inaudi, N. Casanova, P. Kronenberg, S. Vurpillot, Railway bridge monitoring during construction and sliding, SPIE Conference on Smart Structures and Materials, March 5-6, 1997, San Diego, USA.

[23] D. Sackin, J. Garrett, K. J. Gabriel, M. E. Patton, Embedded Microdevices for Infrastructure Monitoring, Proceedings of Annual Congress of the International Association for Bridge and Structural Engineers (IABSE), September, 2000.

[24] C.M. Eastman, A. Siabiris, A generic building product model incorporating building type information, Automation in Construction, Vol. 3 (4), Elsevier, New York, USA, 1995, pp. 283-304.

[25] H.C. Howard, J.A. Abdalla, D.H. Phan, Primitive-composite approach for structural data modeling, Journal of Computing in Civil Engineering, vol. 6 (1), ASCE, New York, USA, 1992, pp. 19-40.

[26] G.A. Van Nederveen, F.P. Tolman, Modeling multiple views on buildings, Automation in Construction, vol. 1 (3), Elsevier, New York, USA, 1992, pp. 215-224.

[27] M. Fischer, F. Aalami, Scheduling with computer-interpretable construction method models. Journal of Construction Engineering and Management, Vol. 122 (4), ASCE, New York, USA, 1996, pp. 337-47.

[28] R. Akbas, M. Fischer, Examples of product model transformations in construction, 8DBMC, May 30 - June 3, 1999, Vancouver, BC, Canada, vol. 4, Information Technology in Construction, CIB W78 Workshop, NRC Research Press, Ottawa, pp. 2737-2746.

[29] R. Stouffs, R. Krishnamurti, SORTS: A concept for representational flexibility, CAAD Futures, R. Junge (Ed.), Kluwer Academic, Dordrecht, The Netherlands, 1997, pp. 553564.

[30] R. Stouffs, R. Krishnamurti, Representational flexibility for design, Artificial Intelligence in Design 2002, JS Gero (Ed.), Kluwer Academic, Dordrecht, The Netherlands, 2002, pp. 129-139 
[31] R. Stouffs, R. Krishnamurti, Data views, data recognition, design queries and design rules. First International Conference on Design Computing and Cognition. July 19-21 2004, MIT, Cambridge, USA.

[32] F. Boukamp, B. Akinci, Towards automated defect detection: Object-oriented modeling of construction specifications, ICCCBE, Weimar, Germany, June 2004, on CD.

[33] C. Gordon, B. Akinci, J. Garrett, An Approach for Planning Sensor-Based Inspection of the Built Environment, ICCCBE, Weimar, Germany, June 2004, on CD.

[34] E. Latimer, D. Latimer, R. Saxena, C. Lyons, L. Michaux-Smith, S. Thayer, Sensor space planning with applications to construction environments, Proceedings of the IEEE International Conference on Robotics and Automation, New Orleans, LA, USA, April 26May 1, 2004, pp. 4454-4460.

[35] C. Gordon, F. Boukamp, D. Huber, E. Latimer, K. Park, B. Akinci, Combining reality capture technologies for construction defect detection: A case study, EIA9: E-Activities and Intelligent Support in Design and the Built Environment, 9th EuropIA International Conference, Istanbul, Turkey, 8-10 October 2003, pp. 99-108.

[36] M. Wheeler, Automatic modeling and localization for object recognition, $\mathrm{PhD}$. Thesis, Robotics Institute, Carnegie Mellon University, 1996.

[37] Robert Bergevin and Marc Soucy and Herve Gagnon and Denis Laurendeau, Towards a general multi-view registration technique, IEEE Transactions on Pattern Analysis and Machine Intelligence, May 1996, vol. 18 (5), pp. 540-547.

[38] Raindrop Geomagic, Inc., www.geomagic.com, accessed June 2004.

[39] D. Huber, M. Hebert, Fully automatic registration of multiple 3D data sets, Image and Vision Computing, vol. 21 (7), July 2003, pp. 637-650.

[40] F. Bernardini, H. Rushmeier, Strategies for registering range images from unknown camera positions, Proceedings of Three-Dimensional Image Capture and Applications III, San Jose, CA, USA, January 2000, vol. 3958 of Proceedings of SPIE, pp. 200-206.

[41] P. Neugebauer, Reconstruction of real-world objects via simultaneous registration and robust combination of multiple range images, International Journal of Shape Modeling, 1997, vol. 3 (1-2), pp.71-90.

[42] R. Benjemaa, F. Schmitt, A solution for the registration of multiple 3D point sets using unit quaternions, Proceedings of the 5th European Conference on Computer Vision (ECCV 1998), Freiburg, Germany, June 1998, pp. 34-50.

[43] Y. Chen, G. Medioni, Object modeling by registration of multiple range images, Image and Vision Computing, April 1992, vol. 10 (3) pp. 145-155.

[44] C. Chua, R. Jarvis, Point signatures: a new representation for $\{3 D\}$ object recognition, International Journal of Computer Vision, October 1997, vol. 25 (1), pp. 63-85.

[45] A. Johnson, M. Hebert, Using spin images for efficient object recognition in cluttered 3D scenes, IEEE Transactions on Pattern Analysis and Machine Intelligence, May 1999, vol. 21 (5), pp.433-449.

[46] C. Gordon and B. Akinci, Technology and Process Assessment of Using LADAR and Embedded Sensing for Construction Quality Control, Construction Research Congress 2005, San Diego, CA, April 5-7, 2005.

[47] ACI, Building Code Requirements for Structural Concrete (ACI 318-02)and Commentary (ACI 318R-02), American Concrete Institute, Reported By ACI Committee 318, 2002.

[48] ACI, Guide to Formwork for Concrete (ACI 347-01), American Concrete Institute, Reported By ACI Committee 347, 2001. 